\title{
A note on degenerate Bernstein polynomials
}

Taekyun Kim', Dae San Kim², Gwan-Woo Jang ${ }^{1}$ and Jongkyum Kwon ${ }^{3 *}$

${ }^{*}$ Correspondence:

mathkjk26@gnu.ac.kr

${ }^{3}$ Department of Mathematics

Education and ERI, Gyeongsang

National University, Jinju, Republic of Korea

Full list of author information is available at the end of the article

\begin{abstract}
Recently, degenerate Bernstein polynomials have been introduced by Kim and Kim. In this paper, we investigate some properties and identities for the degenerate Bernstein polynomials associated with special numbers and polynomials including degenerate Bernstein polynomials and central factorial numbers of the second kind.
\end{abstract}

MSC: $11 \mathrm{~B} 75 ; 11 \mathrm{~B} 83$

Keywords: Degenerate Bernstein polynomials; Degenerate Bernoulli polynomials; Central factorial numbers of the second kind

\section{Introduction}

Bernstein polynomials were first used by Bernstein in a constructive proof for the StoneWeierstrass approximation theorem (see [2, 6, 21]). With the advent of computer graphics, Bernstein polynomials, restricted to the interval $[0,1]$, became important in the form of Bézier curves (see [6]). The Bernstein polynomials are the mathematical basis for Bézier curves, which are frequently used in the mathematical field of numerical analysis (see [6, 24]). The study of degenerate versions of special numbers and polynomials began with the papers by Carlitz (see [3, 4]). Kim and his research colleagues have been studying various degenerate numbers and polynomials by means of generating functions, Fourier series, combinatorial methods, umbral calculus, $p$-adic analysis, and differential equations (see $[10,11,13,17-19])$.

As a degenerate version of Bernstein polynomials, the degenerate Bernstein polynomials were introduced recently (see (1.9)). Here we will study for the degenerate Bernstein polynomials some fundamental properties and identities associated with special numbers and polynomials including degenerate Bernoulli polynomials and central factorial numbers of the second kind. Also, in the last section we will consider a matrix representation for those polynomials. For some recent works related to the present paper, the reader may want to see $[14,20,22,25,27,29]$. The rest of this section is devoted to reviewing what we need in the following sections.

For $k, n \in \mathbb{Z}_{\geq 0}$, the Bernstein polynomials of degree $n$ are defined by

$$
B_{k, n}(x)=\left(\begin{array}{l}
n \\
k
\end{array}\right) x^{k}(1-x)^{n-k} \quad(\text { see }[1,2,6,21,26,28])
$$

where $x \in[0,1]$. 
For $\lambda \in \mathbb{R}$, the degenerate Bernoulli polynomials of order $r$ are defined by the generating function

$$
\left(\frac{t}{(1+\lambda t)^{\frac{1}{\lambda}}-1}\right)^{r}(1+\lambda t)^{\frac{x}{\lambda}}=\sum_{n=0}^{\infty} \beta_{n, \lambda}^{(r)}(x) \frac{t^{n}}{n !}
$$

When $r=1, \beta_{n, \lambda}(x)=\beta_{n, \lambda}^{(1)}(x)(n \geq 0)$ are called the degenerate Bernoulli polynomials (see $[3,4])$. Further, $\beta_{n, \lambda}=\beta_{n, \lambda}(0)$ are called the degenerate Bernoulli numbers.

The falling factorial sequences are defined by

$$
(x)_{0}=1, \quad(x)_{n}=x(x-1)(x-2) \cdots(x-n+1) \quad(n \geq 1) \quad(\text { see }[15,23]) .
$$

The $\lambda$-analogue of the falling factorial sequences are given by

$$
(x)_{0, \lambda}=1, \quad(x)_{n, \lambda}=x(x-\lambda)(x-2 \lambda) \cdots(x-(n-1) \lambda) \quad(n \geq 1) \quad(\text { see }[7,15,16]) .
$$

Note that $\lim _{\lambda \rightarrow 1}(x)_{n, \lambda}=(x)_{n}, \lim _{\lambda \rightarrow 0}(x)_{n, \lambda}=x^{n}$.

It is known that the degenerate exponential function is defined by

$$
(1+\lambda t)^{\frac{x}{\lambda}}=\sum_{n=0}^{\infty}(x)_{n, \lambda} \frac{t^{n}}{n !} \quad(\text { see }[16])
$$

The $\lambda$-binomial coefficients are given by

$$
\left(\begin{array}{l}
x \\
n
\end{array}\right)_{\lambda}=\frac{(x)_{n, \lambda}}{n !}=\frac{x(x-\lambda)(x-2 \lambda) \cdots(x-(n-1) \lambda)}{n !} \quad(\text { see }[8,16]) .
$$

From (1.6), we have

$$
\left(\begin{array}{c}
x+y \\
n
\end{array}\right)_{\lambda}=\sum_{l=0}^{n}\left(\begin{array}{l}
x \\
l
\end{array}\right)_{\lambda}\left(\begin{array}{c}
y \\
n-l
\end{array}\right)_{\lambda} \quad(\text { see }[8,12])
$$

which is equivalent to

$$
(x+y)_{n, \lambda}=\sum_{l=0}^{n}\left(\begin{array}{l}
n \\
l
\end{array}\right)(x)_{l, \lambda}(y)_{n-l, \lambda} \quad(\text { see }[12,14]) .
$$

Recently, Kim and Kim $[12,14]$ introduced the degenerate Bernstein polynomials of degree $n, B_{k, n}(x \mid \lambda)(n, k \geq 0)$, which are given by

$$
\frac{(x)_{k, \lambda}}{k !} t^{k}(1+\lambda t)^{\frac{1-x}{\lambda}}=\sum_{n=k}^{\infty} B_{k, n}(x \mid \lambda) \frac{t^{n}}{n !} \quad(\text { see }[12])
$$

where $k$ is a nonnegative integer. 
From (1.9), we note that

$$
B_{k, n}(x \mid \lambda)=\left(\begin{array}{l}
n \\
k
\end{array}\right)(x)_{k, \lambda}(1-x)_{n-k, \lambda} \quad(n \geq k \geq 0) \quad(\text { see }[12]) .
$$

Thus, by (1.10), we easily get

$$
(x)_{i, \lambda}=\frac{1}{(1-\lambda i)_{n-i, \lambda}} \sum_{k=i}^{n} \frac{\left(\begin{array}{c}
k \\
i
\end{array}\right)}{\left(\begin{array}{l}
n \\
i
\end{array}\right)} B_{k, n}(x \mid \lambda) \quad(\text { see [12]), }
$$

where $i, n \in \mathbb{N}$ with $i \leq n, x \in[0,1]$.

As is well known, the Stirling numbers of the second kind are defined by

$$
x^{n}=\sum_{l=0}^{n} S_{2}(n, l)(x)_{l} \quad(n \geq 0) \quad(\text { see }[7,12]) .
$$

In [8], the degenerate Stirling numbers of the second kind are given by

$$
(x)_{n, \lambda}=\sum_{l=0}^{n} S_{2, \lambda}(n, l)(x)_{l} \quad(n \geq 0)
$$

Note that $\lim _{\lambda \rightarrow 0} S_{2, \lambda}(n, l)=S_{2}(n, l)$.

The degenerate Bernstein polynomials have been introduced recently by Kim and Kim. In this paper, we investigate some properties and identities for the degenerate Bernstein polynomials associated with special numbers and polynomials including degenerate Bernstein polynomials and central factorial numbers of the second kind.

\section{Some fundamental properties of the degenerate Bernstein polynomials}

First, we observe that

$$
\begin{aligned}
(1 & -x-(n-k-1) \lambda) B_{k, n-1}(x \mid \lambda)+(x-(k-1) \lambda) B_{k-1, n-1}(x \mid \lambda) \\
& =\left(\begin{array}{c}
n-1 \\
k
\end{array}\right)(x)_{k, \lambda}(1-x)_{n-k, \lambda}+\left(\begin{array}{l}
n-1 \\
k-1
\end{array}\right)(x)_{k, \lambda}(1-x)_{n-k, \lambda} \\
& =\left[\left(\begin{array}{c}
n-1 \\
k
\end{array}\right)+\left(\begin{array}{c}
n-1 \\
k-1
\end{array}\right)\right](x)_{k, \lambda}(1-x)_{n-k, \lambda} \\
& =\left(\begin{array}{c}
n \\
k
\end{array}\right)(x)_{k, \lambda}(1-x)_{n-k, \lambda}=B_{k, n}(x \mid \lambda) \quad(n, k \in \mathbb{N}) .
\end{aligned}
$$

Thus, by (2.1), we get the next theorem which already appeared in [12].

Theorem 2.1 For $n, k \in \mathbb{N}$, we have

$$
\begin{aligned}
& (1-x-(n-k-1) \lambda) B_{k, n-1}(x \mid \lambda)+(x-(k-1) \lambda) B_{k-1, n-1}(x \mid \lambda) \\
& \quad=B_{k, n}(x \mid \lambda) .
\end{aligned}
$$

By (1.8) and (1.10), we easily get

$$
\sum_{i=0}^{k} B_{i, k}(x \mid \lambda)=\sum_{i=0}^{k}\left(\begin{array}{l}
k \\
i
\end{array}\right)(x)_{i, \lambda}(1-x)_{k-i, \lambda}=(x+1-x)_{k, \lambda}=(1)_{k, \lambda}
$$


where $k$ is a nonnegative integer.

From (1.10), we have

$$
\begin{aligned}
(x-i \lambda) B_{i, n}(x \mid \lambda) & =(x-i \lambda)\left(\begin{array}{c}
n \\
i
\end{array}\right)(x)_{i, \lambda}(1-x)_{n-i, \lambda} \\
& =\left(\begin{array}{c}
n \\
i
\end{array}\right)(x)_{i+1, \lambda}(1-x)_{n-i, \lambda} \\
& =\frac{\left(\begin{array}{c}
n \\
i
\end{array}\right)}{\left(\begin{array}{c}
n+1 \\
i+1
\end{array}\right)}\left(\begin{array}{c}
n+1 \\
i+1
\end{array}\right)(x)_{i+1, \lambda}(1-x)_{n+1-(i+1), \lambda} \\
& =\frac{\left(\begin{array}{c}
n \\
i
\end{array}\right)}{\left(\begin{array}{c}
n+1 \\
i+1
\end{array}\right)} B_{i+1, n+1}(x \mid \lambda) .
\end{aligned}
$$

Hence, by (2.2) and (2.4), we get the following theorem.

Theorem 2.2 For $k \geq 0$, we have

$$
\begin{aligned}
& \sum_{i=0}^{k} B_{i, k}(x \mid \lambda)=\sum_{i=0}^{k}\left(\begin{array}{c}
k \\
i
\end{array}\right)(x)_{i, \lambda}(1-x)_{k-i, \lambda}=(x+1-x)_{k, \lambda}=(1)_{k, \lambda}, \\
& (x-i \lambda) B_{i, n}(x \mid \lambda)=\frac{i+1}{n+1} B_{i+1, n+1}(x \mid \lambda) .
\end{aligned}
$$

On the other hand, we have

$$
\begin{aligned}
(1 & -x-(n-i) \lambda) B_{i, n}(x \mid \lambda) \\
& =\left(\begin{array}{c}
n \\
i
\end{array}\right)(x)_{i, \lambda}(1-x)_{n-i, \lambda}(1-x-(n-i) \lambda) \\
& =\left(\begin{array}{c}
n \\
i
\end{array}\right)(x)_{i, \lambda}(1-x)_{n+1-i, \lambda}=\frac{\left(\begin{array}{c}
n \\
i
\end{array}\right)}{\left(\begin{array}{c}
n+1 \\
i
\end{array}\right)}\left(\begin{array}{c}
n+1 \\
i
\end{array}\right)(x)_{i, \lambda}(1-x)_{n+1-i, \lambda} \\
& =\frac{\left(\begin{array}{c}
n \\
i
\end{array}\right)}{\left(\begin{array}{c}
n+1 \\
i
\end{array}\right)} B_{i, n+1}(x \mid \lambda) \quad(i, n \in \mathbb{N}) .
\end{aligned}
$$

Thus, by (2.5), we get the next result.

Theorem 2.3 For $0 \leq i \leq n+1$, we have

$$
(1-x-(n-i) \lambda) B_{i, n}(x \mid \lambda)=\frac{n+1-i}{n+1} B_{i, n+1}(x \mid \lambda) .
$$


We observe that

$$
\begin{aligned}
\frac{1}{\left(\begin{array}{c}
n \\
i
\end{array}\right)} & B_{i, n}(x \mid \lambda)+\frac{1}{\left(\begin{array}{c}
n \\
i+1
\end{array}\right)} B_{i+1, n}(x \mid \lambda) \\
& =(x)_{i, \lambda}(1-x)_{n-i, \lambda}+(x)_{i+1, \lambda}(1-x)_{n-i-1, \lambda} \\
& =(x)_{i, \lambda}(1-x)_{n-i-1, \lambda}(1-x-(n-i-1) \lambda+x-i \lambda) \\
& =(x)_{i, \lambda}(1-x)_{n-i-1, \lambda}(1-(n-1) \lambda) \\
& =\frac{1-(n-1) \lambda}{\left(\begin{array}{c}
n-1 \\
i
\end{array}\right)}\left(\begin{array}{c}
n-1 \\
i
\end{array}\right)(x)_{i, \lambda}(1-x)_{n-i-1, \lambda} \\
& =\frac{1-\left(\begin{array}{c}
n-1) \lambda \\
\left(\begin{array}{c}
n-1 \\
i
\end{array}\right)
\end{array}\right.}{} B_{i, n-1}(x \mid \lambda) .
\end{aligned}
$$

Hence, by (2.6), we obtain the following identity which appeared already in [12].

$$
\begin{aligned}
(1-(n-1) \lambda) B_{i, n-1}(x \mid \lambda) & =\left(\begin{array}{c}
n-1 \\
i
\end{array}\right)\left[\frac{B_{i, n}(x \mid \lambda)}{\left(\begin{array}{c}
n \\
i
\end{array}\right)}+\frac{B_{i+1, n}(x \mid \lambda)}{\left(\begin{array}{c}
n \\
i+1
\end{array}\right)}\right] \\
& =\frac{n-i}{n} B_{i, n}(x \mid \lambda)+\frac{i+1}{n} B_{i+1, n}(x \mid \lambda) \quad(n \in \mathbb{N}, i \geq 0) .
\end{aligned}
$$

Also, from (1.1) and (1.10), we have

$$
\frac{B_{k, n}(x \mid \lambda)}{B_{k, n}(x)}=\prod_{l=0}^{k-1}\left(1-\lambda \frac{l}{x}\right) \prod_{l=0}^{n-k-1}\left(1-\lambda \frac{l}{1-x}\right) .
$$

Hence, by (2.7) and (2.8), we get the following theorem.

Theorem 2.4 For $n, k, i \in \mathbb{N}$ with $i \leq n$ and $k \leq n$, we have

$$
\begin{aligned}
& (1-(n-1) \lambda) B_{i, n-1}(x \mid \lambda)=\frac{n-i}{n} B_{i, n}(x \mid \lambda)+\frac{i+1}{n} B_{i+1, n}(x \mid \lambda), \\
& \frac{B_{k, n}(x \mid \lambda)}{B_{k, n}(x)}=\prod_{l=0}^{k-1}\left(1-\lambda \frac{l}{x}\right) \prod_{l=0}^{n-k-1}\left(1-\lambda \frac{l}{1-x}\right) .
\end{aligned}
$$

\section{Some identities for degenerate Bernstein polynomials associated with special numbers and polynomials}

Here in this section, we are going to derive some identities associated with special numbers and polynomials including the degenerate Bernoulli polynomials and central factorial numbers of the second kind.

From (1.2), we note that

$$
\frac{t}{(1+\lambda t)^{\frac{1}{\lambda}}-1}=\sum_{n=0}^{\infty} \beta_{n, \lambda} \frac{t^{n}}{n !}
$$

and

$$
\beta_{n, \lambda}(x)=\sum_{l=0}^{n}\left(\begin{array}{l}
n \\
l
\end{array}\right)(x)_{n-l, \lambda} \beta_{l, \lambda} \quad(\text { see }[3,4]) .
$$


Thus, by (3.1), we get

$$
\begin{aligned}
t & =\left(\sum_{l=0}^{\infty} \beta_{l, \lambda} \frac{t^{l}}{l !}\right)\left(\sum_{m=0}^{\infty}(1)_{m, \lambda} \frac{t^{m}}{m !}-1\right) \\
& =\sum_{n=0}^{\infty}\left(\sum_{l=0}^{n}\left(\begin{array}{l}
n \\
l
\end{array}\right)(1)_{n-l, \lambda} \beta_{l, \lambda}-\beta_{n, \lambda}\right) \frac{t^{n}}{n !} .
\end{aligned}
$$

By comparing the coefficients on both sides of (3.2), we get

$$
\sum_{l=0}^{n}\left(\begin{array}{l}
n \\
l
\end{array}\right)(1)_{n-l, \lambda} \beta_{l, \lambda}-\beta_{n, \lambda}=\left\{\begin{array}{ll}
1, & \text { if } n=1, \\
0, & \text { if } n>1,
\end{array} \quad \beta_{0, \lambda}=1 \quad(\text { see }[3,4]) .\right.
$$

When $n=1$, we have

$$
\beta_{1, \lambda}(1)=\sum_{l=0}^{1}\left(\begin{array}{l}
1 \\
l
\end{array}\right)(1)_{1-l, \lambda} \beta_{l, \lambda}=1+\beta_{1, \lambda}
$$

By (1.2), we easily get

$$
\begin{aligned}
\sum_{n=0}^{\infty} \beta_{n, \lambda}(1-x) \frac{t^{n}}{n !} & =\frac{t}{(1+\lambda t)^{\frac{1}{\lambda}}-1}(1+\lambda t)^{\frac{1-x}{\lambda}} \\
& =\frac{-t}{(1+(-\lambda)(-t))^{-\frac{1}{\lambda}}-1}(1+(-\lambda)(-t))^{-\frac{x}{\lambda}} \\
& =\sum_{n=0}^{\infty} \beta_{n,-\lambda}(x)(-1)^{n} \frac{t^{n}}{n !} .
\end{aligned}
$$

Comparing the coefficients on both sides of (3.4), we have

$$
\beta_{n, \lambda}(1-x)=(-1)^{n} \beta_{n,-\lambda}(x) \quad(n \geq 0) .
$$

Taking $x=-1, \beta_{n, \lambda}(2)=(-1)^{n} \beta_{n,-\lambda}(-1)(n \geq 0)$. We observe that

$$
\begin{aligned}
\sum_{n=0}^{\infty} \beta_{n, \lambda}(2) \frac{t^{n}}{n !} & =\frac{t}{(1+\lambda t)^{\frac{1}{\lambda}}-1}(1+\lambda t)^{\frac{2}{\lambda}} \\
& =\frac{t}{(1+\lambda t)^{\frac{1}{\lambda}}-1}\left((1+\lambda t)^{\frac{1}{\lambda}}-1+1\right)(1+\lambda t)^{\frac{1}{\lambda}} \\
& =t(1+\lambda t)^{\frac{1}{\lambda}}+\frac{t}{(1+\lambda t)^{\frac{1}{\lambda}}-1}(1+\lambda t)^{\frac{1}{\lambda}} \\
& =t \sum_{n=0}^{\infty}(1)_{n, \lambda} \frac{t^{n}}{n !}+\sum_{n=0}^{\infty} \beta_{n, \lambda}(1) \frac{t^{n}}{n !} \\
& =\sum_{n=0}^{\infty}\left(n(1)_{n-1, \lambda}+\beta_{n, \lambda}(1)\right) \frac{t^{n}}{n !}
\end{aligned}
$$


By (3.5), we get

$$
\beta_{n, \lambda}(2)=n(1)_{n-1, \lambda}+\beta_{n, \lambda}(1) \quad(n \geq 1) .
$$

It is not difficult to show that

$$
\begin{aligned}
\frac{t}{(1+\lambda t)^{\frac{1}{\lambda}}-1}(1+\lambda t)^{\frac{x}{\lambda}} \\
=\frac{t}{(1+\lambda t)^{\frac{1}{\lambda}}-1}(1+\lambda t)^{\frac{x-1}{\lambda}}\left((1+\lambda t)^{\frac{1}{\lambda}}-1+1\right) \\
=t(1+\lambda t)^{\frac{x-1}{\lambda}}+\frac{t}{(1+\lambda t)^{\frac{1}{\lambda}}-1}(1+\lambda t)^{\frac{x-1}{\lambda}} \\
=t(1+\lambda t)^{\frac{x-1}{\lambda}}+t(1+\lambda t)^{\frac{x-2}{\lambda}}+\frac{t}{(1+\lambda t)^{\frac{1}{\lambda}}-1}(1+\lambda t)^{\frac{x-2}{\lambda}} \\
=t(1+\lambda t)^{\frac{x-1}{\lambda}}+t(1+\lambda t)^{\frac{x-2}{\lambda}}+t(1+\lambda t)^{\frac{x-3}{\lambda}}+\frac{t}{(1+\lambda t)^{\frac{1}{\lambda}}-1} \\
\quad \times(1+\lambda t)^{\frac{x-3}{\lambda}} .
\end{aligned}
$$

Continuing this process, we have

$$
\begin{aligned}
\sum_{n=0}^{\infty} \beta_{n, \lambda}(x) \frac{t^{n}}{n !} & =t \sum_{i=1}^{k}(1+\lambda t)^{\frac{x-i}{\lambda}}+\frac{t}{(1+\lambda t)^{\frac{1}{\lambda}}-1}(1+\lambda t)^{\frac{x-k}{\lambda}} \\
& =t \sum_{i=1}^{k} \sum_{n=0}^{\infty}(x-i)_{n, \lambda} \frac{t^{n}}{n !}+\sum_{n=0}^{\infty} \beta_{n, \lambda}(x-k) \frac{t^{n}}{n !} \\
& =\sum_{n=1}^{\infty}\left(\sum_{i=1}^{k} n(x-i)_{n-1, \lambda}\right) \frac{t^{n}}{n !}+\sum_{n=0}^{\infty} \beta_{n, \lambda}(x-k) \frac{t^{n}}{n !} .
\end{aligned}
$$

Therefore, by comparing the coefficients on both sides of (3.8), we obtain the following proposition.

Proposition 1 For $k \in \mathbb{N}$ and $n \geq 0$, we have

$$
\beta_{n, \lambda}(x)=\sum_{i=1}^{k} n(x-i)_{n-1, \lambda}+\beta_{n, \lambda}(x-k) .
$$

In particular,

$$
\beta_{n, \lambda}(k)=\sum_{i=1}^{k} n(k-i)_{n-1, \lambda}+\beta_{n, \lambda} .
$$


From (1.9), we have

$$
\begin{aligned}
(x)_{k, \lambda}(1+\lambda t)^{\frac{1-x}{\lambda}} & =\frac{k !}{t^{k}} \frac{1}{k !}(x)_{k, \lambda} t^{k}(1+\lambda t)^{\frac{1-x}{\lambda}} \\
& =\frac{k !}{t^{k}} \sum_{n=k}^{\infty} B_{k, n}(x \mid \lambda) \frac{t^{n}}{n !}=k ! \sum_{n=0}^{\infty} B_{k, n+k}(x \mid \lambda) \frac{n !}{(n+k) !} \frac{t^{n}}{n !} \\
& =\sum_{n=0}^{\infty} \frac{1}{\left(\begin{array}{c}
n+k \\
k
\end{array}\right)} B_{k, n+k}(x \mid \lambda) \frac{t^{n}}{n !} .
\end{aligned}
$$

On the other hand,

$$
(x)_{k, \lambda}(1+\lambda t)^{\frac{1-x}{\lambda}}=\sum_{n=0}^{\infty}(x)_{k, \lambda}(1-x)_{n, \lambda} \frac{t^{n}}{n !} .
$$

From (3.9) and (3.10), we have

$$
(x)_{k, \lambda}(1-x)_{n, \lambda}=\frac{1}{\left(\begin{array}{c}
n+k \\
k
\end{array}\right)} B_{k, n+k}(x \mid \lambda) \quad(n, k \geq 0) .
$$

Note here that (3.11) also follows from (1.10) by replacing $n$ by $n+k$.

From (1.2), we note that

$$
\begin{aligned}
(x)_{k, \lambda}(1+\lambda t)^{\frac{1-x}{\lambda}} & =\frac{(x)_{k, \lambda}}{t} \frac{t}{(1+\lambda t)^{\frac{1}{\lambda}}-1}(1+\lambda t)^{\frac{1-x}{\lambda}}\left((1+\lambda t)^{\frac{1}{\lambda}}-1\right) \\
& =\frac{(x)_{k, \lambda}}{t}\left\{\frac{t}{(1+\lambda t)^{\frac{1}{\lambda}}-1}(1+\lambda t)^{\frac{2-x}{\lambda}}-\frac{t}{(1+\lambda t)^{\frac{1}{\lambda}}-1}(1+\lambda t)^{\frac{1-x}{\lambda}}\right\} \\
& =\frac{(x)_{k, \lambda}}{t}\left\{\sum_{n=1}^{\infty} \beta_{n, \lambda}(2-x)-\beta_{n, \lambda}(1-x)\right\} \frac{t^{n}}{n !} \\
& =(x)_{k, \lambda} \sum_{n=0}^{\infty}\left(\frac{\beta_{n+1, \lambda}(2-x)-\beta_{n+1, \lambda}(1-x)}{n+1}\right) \frac{t^{n}}{n !} .
\end{aligned}
$$

By (3.12), we get

$$
(x)_{k, \lambda}(1-x)_{n, \lambda}=(x)_{k, \lambda} \frac{\beta_{n+1, \lambda}(2-x)-\beta_{n+1, \lambda}(1-x)}{n+1},
$$

where $k \in \mathbb{N}$ and $n \geq 0$.

From (1.10) and (3.13), we have

$$
B_{k, n+k}(x \mid \lambda)=\left(\begin{array}{c}
n+k \\
k
\end{array}\right)(x)_{k, \lambda} \frac{\beta_{n+1, \lambda}(2-x)-\beta_{n+1, \lambda}(1-x)}{n+1} .
$$

Theorem 3.1 For $k \in \mathbb{N}$ and $n \geq 0$, we have

$$
B_{k, n+k}(x \mid \lambda)=\left(\begin{array}{c}
n+k \\
k
\end{array}\right)(x)_{k, \lambda} \frac{\beta_{n+1, \lambda}(2-x)-\beta_{n+1, \lambda}(1-x)}{n+1} .
$$


As is well known, the central factorial numbers of the second kind are defined by the generating function

$$
\frac{1}{k !}\left(e^{\frac{1}{2} t}-e^{-\frac{1}{2} t}\right)^{k}=\sum_{n=k}^{\infty} T(n, k) \frac{t^{n}}{n !} \quad(k \geq 0) \quad(\text { see }[5,9]) .
$$

Recently, the degenerate central factorial numbers $T_{\lambda}(n, k)$ of the second kind were introduced by the generating function

$$
\frac{1}{k !}\left((1+\lambda t)^{\frac{1}{2 \lambda}}-(1+\lambda t)^{-\frac{1}{2 \lambda}}\right)^{k}=\sum_{n=k}^{\infty} T_{\lambda}(n, k) \frac{t^{n}}{n !} \quad(\text { see }[16]) .
$$

By (3.15), we get

$$
\begin{aligned}
\sum_{n=k}^{\infty} \lim _{\lambda \rightarrow 0} T_{\lambda}(n, k) \frac{t^{n}}{n !} & =\lim _{\lambda \rightarrow 0} \frac{1}{k !}\left((1+\lambda t)^{\frac{1}{2 \lambda}}-(1+\lambda t)^{-\frac{1}{2 \lambda}}\right)^{k} \\
& =\frac{1}{k !}\left(e^{\frac{1}{2} t}-e^{-\frac{1}{2} t}\right)^{k}=\sum_{n=k}^{\infty} T(n, k) \frac{t^{n}}{n !} .
\end{aligned}
$$

Thus, by (3.16), we get

$$
\lim _{\lambda \rightarrow 0} T_{\lambda}(n, k)=T(n, k) \quad(n, k \geq 0) \quad(\text { see }[9]) .
$$

Now, we observe that

$$
\begin{aligned}
\frac{(x)_{k, \lambda}}{k !} t^{k}(1+\lambda t)^{\frac{1-x}{\lambda}}= & \frac{(x)_{k, \lambda}}{k !}\left((1+\lambda t)^{\frac{1}{2 \lambda}}-(1+\lambda t)^{-\frac{1}{2 \lambda}}\right)^{k} \\
& \times\left(\frac{t}{(1+\lambda t)^{\frac{1}{\lambda}}-1}\right)^{k}(1+\lambda t)^{\frac{1-x+\frac{k}{2}}{\lambda}} \\
& =(x)_{k, \lambda}\left(\sum_{m=k}^{\infty} T_{\lambda}(m, k) \frac{t^{m}}{m !}\right)\left(\sum_{l=0}^{\infty} \beta_{l, \lambda}^{(k)}\left(1-x+\frac{k}{2}\right) \frac{t^{l}}{l !}\right) \\
& =\sum_{n=k}^{\infty}(x)_{k, \lambda} \sum_{m=k}^{n}\left(\begin{array}{c}
n \\
m
\end{array}\right) T_{\lambda}(m, k) \beta_{n-m, \lambda}^{(k)}\left(1-x+\frac{k}{2}\right) \frac{t^{n}}{n !} .
\end{aligned}
$$

By combining the right-hand side of (1.9) with (3.17), we obtain the following theorem.

Theorem 3.2 For $n, k \in \mathbb{N} \cup\{0\}$ with $n \geq k$, we have

$$
B_{k, n}(x \mid \lambda)=(x)_{k, \lambda} \sum_{m=k}^{n}\left(\begin{array}{l}
n \\
m
\end{array}\right) T_{\lambda}(m, k) \beta_{n-m, \lambda}^{(k)}\left(1-x+\frac{k}{2}\right) .
$$

\section{A matrix representation for degenerate Bernstein polynomials}

For $\lambda \in \mathbb{R}$, let

$$
\mathbb{P}_{n, \lambda}=\left\{p_{\lambda}(x) \mid p_{\lambda}(x)=\sum_{i=0}^{n}(x)_{i, \lambda} C_{i, \lambda} \in \mathbb{R}[x]\right\} .
$$

Then $\mathbb{P}_{n, \lambda}$ is the $n+1$-dimensional vector space over $\mathbb{R}$. 
For $B_{\lambda}(x) \in \mathbb{P}_{n, \lambda}$, we note that $B_{\lambda}(x)$ can be written as a linear combination of degenerate Bernstein basis functions:

$$
\begin{aligned}
B_{\lambda}(x)= & C_{0, \lambda} B_{0, n}(x \mid \lambda)+C_{1, \lambda} B_{1, n}(x \mid \lambda)+C_{2, \lambda} B_{2, n}(x \mid \lambda)+\cdots \\
& +C_{n, \lambda} B_{n, n}(x \mid \lambda)
\end{aligned}
$$

where the constants $C_{i, \lambda}$ depend on $\lambda$ for $i=0,1,2, \ldots, n$.

Equation (4.1) can be written as the dot product of two vectors in the following:

$$
B_{\lambda}(x)=\left(\begin{array}{llll}
B_{0, n}(x \mid \lambda) & B_{1, n}(x \mid \lambda) & \cdots & B_{n, n}(x \mid \lambda)
\end{array}\right)\left(\begin{array}{c}
C_{0, \lambda} \\
C_{1, \lambda} \\
\vdots \\
C_{n, \lambda}
\end{array}\right) .
$$

Now, we can convert (4.2) to

$$
\begin{aligned}
& B_{\lambda}(x)=\left(\begin{array}{llll}
1 & x & \cdots & x^{n}
\end{array}\right) \\
& \times\left(\begin{array}{ccccccc}
b_{0,0}(\lambda) & 0 & 0 & 0 & \cdots & 0 & 0 \\
b_{1,0}(\lambda) & b_{1,1}(\lambda) & b_{1,2}(\lambda) & b_{1,3}(\lambda) & \cdots & b_{1, n-1}(\lambda) & b_{1, n}(\lambda) \\
b_{2,0}(\lambda) & b_{2,1}(\lambda) & b_{2,2}(\lambda) & b_{2,3}(\lambda) & \cdots & b_{2, n-1}(\lambda) & b_{2, n}(\lambda) \\
\vdots & & & & & & \vdots \\
b_{n-1,0}(\lambda) & b_{n-1,1}(\lambda) & b_{n-1,2}(\lambda) & b_{n-1,3}(\lambda) & \cdots & b_{n-1, n-1}(\lambda) & b_{n-1, n}(\lambda) \\
b_{n, 0}(\lambda) & b_{n, 1}(\lambda) & b_{n, 2}(\lambda) & b_{n, 3}(\lambda) & \cdots & b_{n, n-1}(\lambda) & b_{n, n}(\lambda)
\end{array}\right) \\
& \times\left(\begin{array}{c}
C_{0, \lambda} \\
C_{1, \lambda} \\
\vdots \\
C_{n, \lambda}
\end{array}\right),
\end{aligned}
$$

where $b_{i, j}(\lambda)$ are the coefficients of the power basis that are used to determine the respective degenerate Bernstein polynomials.

For example, by (1.1), we get

$$
\begin{aligned}
B_{0,2}(x \mid \lambda) & =\left(\begin{array}{l}
2 \\
0
\end{array}\right)(x)_{0, \lambda}(1-x)_{2, \lambda}=(1-x)(1-x-\lambda)=(1-x)^{2}-\lambda(1-x) \\
& =x^{2}+(\lambda-2) x+1-\lambda \\
B_{1,2}(x \mid \lambda) & =\left(\begin{array}{l}
2 \\
1
\end{array}\right)(x)_{1, \lambda}(1-x)_{1, \lambda}=2 x(1-x)=2 x-2 x^{2} \\
B_{2,2}(x \mid \lambda) & =\left(\begin{array}{l}
2 \\
2
\end{array}\right)(x)_{2, \lambda}(1-x)_{0, \lambda}=x(x-\lambda)=x^{2}-\lambda x .
\end{aligned}
$$


In the quadratic case $(n=2), B_{\lambda}(x)$ can be represented in terms of matrices by

$$
B_{\lambda}(x)=\left(\begin{array}{lll}
1 & x & x^{2}
\end{array}\right)\left(\begin{array}{ccc}
1-\lambda & 0 & 0 \\
\lambda-2 & 2 & -\lambda \\
1 & -2 & 1
\end{array}\right)\left(\begin{array}{l}
C_{0, \lambda} \\
C_{1, \lambda} \\
C_{2, \lambda}
\end{array}\right) .
$$

\section{Conclusions}

In Sect. 2, we investigated some fundamental properties for the degenerate Bernstein polynomials. In Sect. 3, we derived some identities for the degenerate Bernstein polynomials associated with special numbers and polynomials including degenerate Bernoulli polynomials and central factorial numbers of the second kind. In many applications, a matrix formulation for the Bernstein polynomials is useful. So, in Sect. 4, we studied some further properties of the matrix representation for degenerate Bernstein polynomials.

\section{Acknowledgements}

This paper was dedicated to the renowned mathematician Professor Gradimir V. Milovanović on the occasion of his 70th anniversary. Also, we would like to thank the referees whose suggestions and comments helped improve the original manuscript greatly.

\section{Funding}

This work was supported by the National Research Foundation of Korea (NRF) grant funded by the Korea government (MEST) (No. 2017R1E1A1A03070882).

\section{Competing interests}

The authors declare that they have no competing interests.

\section{Authors' contributions}

Conceptualization, TK; Formal analysis, DSK and TK; Investigation, DSK and TK; Methodology, DSK and TK; Project administration, JK and TK; Supervision, DSK and TK; Publication fee payment, JK; Writing-original draft, TK; Writing-review and editing, DSK. All authors contributed equally to the manuscript, read, and approved the final manuscript.

\section{Author details}

'Department of Mathematics, Kwangwoon University, Seoul, Republic of Korea. ${ }^{2}$ Department of Mathematics, Sogang University, Seoul, Republic of Korea. ${ }^{3}$ Department of Mathematics Education and ERI, Gyeongsang National University, Jinju, Republic of Korea.

\section{Publisher's Note}

Springer Nature remains neutral with regard to jurisdictional claims in published maps and institutional affiliations.

Received: 5 February 2019 Accepted: 15 April 2019 Published online: 09 May 2019

\section{References}

1. Acikgoz, M., Araci, S.: On the generating function of the Bernstein polynomials. AIP Conf. Proc. CP1281, 1141-1143 (2010)

2. Bernstein, S.N.: Bernstein's Démonstration du théorème de Weierstrass. Comm. Soc. Math. Charkow Ser. 2 t. 13, 1-2 (1912-1913)

3. Carlitz, L.: A degenerate Staudt-Clausen theorem. Arch. Math. (Basel) 7, 28-33 (1956)

4. Carlitz, L.: Degenerate Stirling, Bernoulli and Eulerian numbers. Util. Math. 15, 51-88 (1979)

5. Comtet, L.: The Art of Finite and Infinite Expansions. Reidel, Dordrecht (1974) Translated from the French by J.W Nienhuys

6. Farouki, R.T.: The Bernstein polynomials basis: a centennial retrospective. Comput. Aided Geom. Des. 29(6), 379-419 (2012)

7. Kim, D.S., Kwon, J., Dolgy, D.V., Kim, T.: On central Fubini polynomials associated with central factorial numbers of the second kind. Proc. Jangjeon Math. Soc. 21(4), 589-598 (2018)

8. Kim, T.: A note on degenerate Stirling polynomials of the second kind. Proc. Jangjeon Math. Soc. 20(3), 319-331 (2017)

9. Kim, T.: A note on central factorial numbers. Proc. Jangjeon Math. Soc. 21(4), 575-588 (2018)

10. Kim, T., Kim, D.S.: Identities involving degenerate Euler numbers and polynomials arising from non-linear differential equations. J. Nonlinear Sci. Appl. 9, 2086-2098 (2016)

11. Kim, T., Kim, D.S.: Identities of symmetry for degenerate Euler polynomials and alternating generalized falling factorial sums. Iran. J. Sci. Technol., Trans. A, Sci. 41, 939-949 (2017)

12. Kim, T., Kim, D.S.: Degenerate Bernstein polynomials. Rev. R. Acad. Cienc. Exactas Fís. Nat., Ser. A Mat. (2018) https://doi.org/10.1007/s13398-018-0594-9 
13. Kim, T., Kim, D.S.: Identities for degenerate Bernoulli polynomials and Korobov polynomials of the first kind. Sci. China Math. (2018). https://doi.org/10.1007/s11425-018-9338-5

14. Kim, T., Kim, D.S.: Some identities on degenerate Bernstein and degenerate Euler polynomials. Mathematics 7(1), Article ID 47 (2019). https://doi.org/10.3390/math7010047

15. Kim, T., Kim, D. S.: Extended stirling numbers of the first kind associated with Daehee numbers and polynomials. Rev. R. Acad. Cienc. Exactas Fís. Nat., Ser. A Mat. 113(2), 1159-1171 (2019)

16. Kim, T., Kim, D.S.: Degenerate central factorial numbers of the second kind. Submitted

17. Kim, T., Kim, D.S., Jang, G.-W.: Differential equations associated with degenerate Cauchy numbers. Iran. J. Sci. Technol., Trans. A, Sci. (2018). https://doi.org/10.1007/s40995-018-0531-y

18. Kim, T., Kim, D.S., Jang, G.-W., Jang, L.-C.: Degenerate ordered Bell numbers and polynomials associated with umbral calculus. J. Nonlinear Sci. Appl. 10, 5142-5155 (2017)

19. Kim, T., Yao, Y., Kim, D.S., Jang, G.-W.: Degenerate $r$-Stirling numbers and $r$-Bell polynomials. Russ. J. Math. Phys. 25(1), 44-58 (2018)

20. Kurt, V.: Some relation between the Bernstein polynomials and second kind Bernoulli polynomials. Adv. Stud. Contemp. Math. (Kyungshang) 23(1), 43-48 (2013)

21. Lorentz, G.G.: Bernstein Polynomials, 2nd edn. Chelsea, New York (1986)

22. Ostrovska, S.: On the 9 -Bernstein polynomials. Adv. Stud. Contemp. Math. (Kyungshang) 11(2), 193-204 (2005)

23. Roman, S.: The Umbral Calculus. Pure and Applied Mathematics, vol. 111. Academic Press, New York (1984)

24. Simsek, B., Yardimci, A.: Using Bezier curves in medical applications. Filomat 30(4), 937-943 (2016)

25. Simsek, Y.: A new class of polynomials associated with Bernstein and beta polynomials. Math. Methods Appl. Sci. 37(5), 676-685 (2014)

26. Simsek, Y:: Analysis of the Bernstein basis functions: an approach to combinatorial sums involving binomial coefficients and Catalan numbers. Math. Methods Appl. Sci. 38(14), 3007-3021 (2015)

27. Simsek, Y.: New families of special numbers for computing negative order Euler numbers and related numbers and polynomials. Appl. Anal. Discrete Math. 12(1), 1-35 (2018)

28. Simsek, Y: Generating functions for the Bernstein type polynomials: a new approach to deriving identities and applications for the polynomials. Hacet. J. Math. Stat. 43(1), 1-14 (2101)

29. Simsek, Y., Bayad, A., Lokesha, V:: $q$-Bernstein polynomials related to $q$-Frobenius-Euler polynomials, I-functions, and q-Stirling numbers. Math. Methods Appl. Sci. 35(8), 877-884 (2012)

\section{Submit your manuscript to a SpringerOpen ${ }^{\circ}$ journal and benefit from:}

- Convenient online submission

- Rigorous peer review

- Open access: articles freely available online

- High visibility within the field

Retaining the copyright to your article

Submit your next manuscript at $\gg$ springeropen.com 